\title{
Research Paper \\ Instantaneous Effect of Insole on the Balance of Adolescents With Flat Foot and Pes Cavus
}

\author{
Ali Fattahi ${ }^{1}$ (), *Zahra Koreili ${ }^{1}$, , Mitra Ameli $^{2}$ 두
}

1. Department of Sports Biomechanics, Faculty of Physical Education and Sports Science, Central Tehran Branch, Islamic Azad University, Tehran, Iran 2. Athletic \& Clinical Research Center, Pouyesh Setaregan Salamat, Tehran, Iran.

\begin{tabular}{|c|c|}
\hline $\begin{array}{l}\text { Use your device to scan } \\
\text { and read the article online }\end{array}$ & \\
\hline 口rming & Ctration: Fattahi A, Koreili Z, Ameli M. [Instantaneous Effect of Insole on the Balance of Adolescents With Flat Foot and Pes \\
\hline yosp & Cavus (Persian)]. Journal of Sport Biomechanics. 2020; 6(1):44-53. https://doi.org/10.32598/biomechanics.6.1.6 \\
\hline It & delishttps://doi.org/10.32598/biomechanics.6.1.6 \\
\hline
\end{tabular}

(c) (i) (5)

Article Info:

Received: 13 Mar 2020

Accepted:10 May 2020

Available Online: 01 Jun 2020

Keywords:

Insole, Balance, Flat

foot, Pes cavus, Ado-

lescents

\section{ABSTRACT}

Objective The purpose of this study was to examine the instantaneous effect of insole on the balance of adolescents with flat foot and Pes Cavus.

Methods In this quasi-experimental study, 34 adolescents with flat foot and Pes Cavus (17 girls and 17 boys) in the age range of 11 to 14 years in Tehran were randomly selected and their balance performance in static and dynamic positions was examined before and after instantaneous using of insole. Data were analyzed using paired t-test and independent t-test.

Results Paired t-test results showed that the instantaneous effect of insole on dynamic balance is significant $(P=0.00)$, but it had no significant effect on static balance $(P=0.07)$. The results of independent t-test showed no significant difference between the two groups in terms of dynamic balance $(P=0.99)$ and static balance $(P=0.52)$ in Pre-test phase. There was no significant difference between the two groups in dynamic balance $(P=0.25)$ and static balance $(P=0.72)$ in Post-test phase, either.

Conclusion Using medical insole can improve the dynamic balance in adolescents with Pes Cavus and flat foot, but has no effect on their static balance.

\section{Extended Abstract}

\section{Introduction}

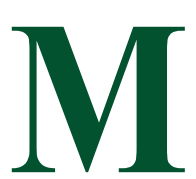

aintaining balance is one of the most basic neuromuscular functions in the most of simple and complex activities. Plantar arches play an important role in regulating and distributing plantar pressure. In the healthy people, the presence of longitudinal and transverse plantar arches, support of bone structures and soft tissue stabilizes the subtalar and midtarsal joints. Having stability in these joints during dynamic activi- ties such as walking provides the necessary force to move forward and transfer that force to the foot. Prefabricated insoles increase balance and reduce extra lateral movements by creating more contact surface and more appropriate pressure distribution over the sole of the foot. The presence of musculoskeletal abnormalities such as flat feet and Pes Cavus and structural features of the foot affect the balance of people. Decreased balance leads to decreased performance and provides the basis for injury, especially in the ankle.

Many studies have been done on static and dynamic balance in people with plantar abnormalities, but little research has been done on the effect of medical insoles on the bal-

\section{* Corresponding Author:}

Zahra Koreili

Address: Department of Sports Biomechanics, Faculty of Physical Education and Sports Science, Central Tehran Branch, Islamic Azad University, Tehran, Iran.

Tel: +98 (912) 5846472

E-mail: zahrakoreili@yahoo.com 
Table 1. Paired t-test results of comparing the instantaneous effect of medical insole on the balance of participants in two groups

\begin{tabular}{|c|c|c|c|c|c|c|}
\hline \multirow{2}{*}{ Group } & \multirow{2}{*}{ Variable } & \multicolumn{2}{|c|}{ Mean $\pm S D$} & \multirow{2}{*}{ Mean Difference } & \multirow{2}{*}{$\mathbf{t}$} & \multirow{2}{*}{$\mathbf{P}$} \\
\hline & & Pre-test & Post-test & & & \\
\hline \multirow{2}{*}{ With Pes Cavus } & Dynamic balance (12) & $65.08 \pm 9.93$ & $83.91 \pm 8.43$ & -18.83 & -7.83 & 0.00 \\
\hline & Static balance (12) & $18.58 \pm 3.42$ & $19.75 \pm 3.93$ & -1.16 & -0.82 & 0.42 \\
\hline \multirow{2}{*}{ With flat feet } & Dynamic balance (22) & $65.04 \pm 12.40$ & $87.31 \pm 7.97$ & -22.27 & -6.37 & 0.00 \\
\hline & Static balance (22) & $17.77 \pm 3.61$ & $19.27 \pm 3.64$ & -1.50 & -1.69 & 0.10 \\
\hline
\end{tabular}

ance of children and adolescents. Since the most daily activities require balance and many injuries occur in static and dynamic positions, the aim of the present study was to evaluate the instantaneous effect of insole on the balance of adolescents with flat foot and Pes Cavus.

\section{Methods}

This is a descriptive quasi-experimental study conducted on 34 adolescents aged 11-14 years old in Tehran (17 girls and 17 boys; who were randomly selected. A wall mounted stadiometer (model $26 \mathrm{SM}$ ) was used to measure the height and a digital scale (BS101) was used to measure the weight in subjects. For assessing their soles, the type of abnormality was determined first by observational method and then by using a foot scanner (3D BodyView, USA). The validity of this device has been calculated by the manufacturer ( $\mathrm{r}=0.64)$. Its intrapersonal and interpersonal reliabilities using intra-class correlation coefficient were obtained 0.62 and 0.91 , respectively. Sharpened Romberg test was used to measure static balance of participants, and ENC device (Danesh Salar Iranian Co., Iran) was used to measure their dynamic balance.

This device has two parts; a special part for standing and the other part to display information related to the balance. The movement of the person on the screen is in the mediallateral direction and according to the visual stimulus. This device has biofeedback and the amplitude of postural stability can be adjusted. It can also be used to measure the balance coordination of body organs. It has a measurement accuracy of one degree with a dimension of $105 \times 82 \mathrm{~cm}$ and the ability to display the percentage of postural balance. The validity of the device has been calculated by the manufacturer using Pearson correlation coefficient between the score of this device and that of Lafayette Manual Muscle Tester $(\mathrm{r}=0.88)$. Its intrapersonal and interpersonal reliabilities using intra-class correlation coefficient were obtained 0.93 and 0.91 , respectively.
Kolmogorov-Smirnov test was used to evaluate the normality of data distribution. Descriptive statistics $(\mathrm{Mean} \pm \mathrm{SD})$ were used to describe variables, paired t-test to investigate the instantaneous effect of the insole, and independent t-test to compare the two groups of flat foot and Pes Cavus at a significance level of 0.05 .

\section{Results}

The mean demographic indicators of the subjects were as follows: the Mean $\pm \mathrm{SD}$ age $=12.23 \pm 1.18$ years, the Mean \pm SD height $=153.47 \pm 10.04 \mathrm{~cm}$, the Mean \pm SD weight $=42.47 \pm 6.23 \mathrm{~kg}$, the Mean $\pm \mathrm{SD}$ of stability index $=93.29 \pm 47.39$ degrees, the Mean \pm SD of right leg length $=24.19 \pm 0.49 \mathrm{~cm}$, and the Mean \pm SD of left leg length $=24.28 \pm 0.46 \mathrm{~cm}$.

The mean of dynamic test score and Sharpened Romberg test score in the post-test phase were higher than those in the pre-test phase. The results of paired t-test showed the significant instantaneous effect of the insole under dynamic balance test $(\mathrm{P}=0.00)$, but no significant difference was observed under Sharpened Romberg test $(\mathrm{P}=0.07)$. The results of paired t-test for instantaneous effect of insole on the two study groups showed that the use of insoles improved the dynamic balance of subjects with Pes Cavus $(\mathrm{P}=0.00)$, but had no significant effect on their static balance $(\mathrm{P}=0.42)$. Using the insoles also improved the dynamic balance of subjects with flat foot $(\mathrm{P}=0.00)$, but had no significant effect on their static balance $(\mathrm{P}=0.10)$ (Table 1).

The results of independent t-test to compare the two groups of flat foot and Pes Cavus, showed no significant difference between the two groups in terms of dynamic balance $(\mathrm{P}=0.99)$ and static balance $(\mathrm{P}=0.52)$ in Pre-test phase. There was no significant difference between the two groups in dynamic balance $(\mathrm{P}=0.25)$ and static balance $(\mathrm{P}=0.72)$ in Post-test phase, either. These results indicate that adolescent girls and boys with flat foot and Pes Cavus had poorer 
dynamic balance before using the insoles. Moreover, the instantaneous effect of medical insole on the dynamic balance of adolescents with flat feet was slightly greater than that of those with Pes Cavus, but medical insole had no significant effect on their static balance.

\section{Conclusion}

The arches of the sole are very influential on the distribution of force and pressure because the weight of the body is distributed by the talus to the front and back of the foot and then transferred to the ground. Deformity in the foot area for any reason causes numerous problems in people's daily living activities such as walking, running, standing, and also in the anatomical structure and arches of the soles and affect the balance of people. Medical insoles can systematically reduce pressure over the foot and help posture stabilization by maintaining the arch of the sole and increasing the plantar surface.

The results of the present study showed that adolescent girls and boys with flat foot and Pes Cavus had poorer dynamic balance before using the insoles. Moreover, the instantaneous effect of medical insole on the dynamic balance of adolescents with flat feet was slightly greater than that of those with Pes Cavus, but medical insole had no significant effect on their static balance. Therefore, it is recommended that people with plantar abnormalities use the mechanical effects of the medical insoles to correct their abnormalities, improve mechanical performance and prevent possible injuries.

\section{Ethical Considerations}

Compliance with ethical guidelines

All ethical principles are considered in this article.

Funding

This research did not receive any grant from funding agencies in the public, commercial, or non-profit sectors.

Authors' contributions

All authors contributed in preparing this article.

Conflicts of interest

The authors declared no conflict of interest.

\section{Acknowledgements}

The authors would like to express their appreciation of all of Athletic \& Clinical Center, Pouyesh Setaregan Salamt, Tehran, Iran. 


\title{
تأثير آنى كفى بر عملكرد تعادلى نوجوانان با عارضه كف ياي صاف و تود
}

\author{
على فتاحى' ه. "زهرا كريلى' ه، ميترا عاملى'ه \\ 1. كروه بيومكانيك ورزشى، دانشكده تربيتبدنى و علوم ورزشى و تربيتبدنى، واحد تهران مركزى، دانشكاه آزاد اسلامى، تهران، ايران.

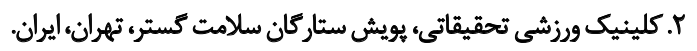

\begin{abstract}
حكيد
هدف هدف مطالعه حاضر بررسى ثأثير آنى كفى بر عملكرد تعادلى نوجوانان با عارضه كف ياى صاف و كود بود.

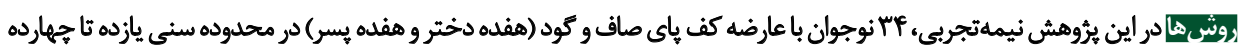

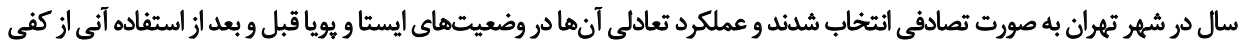

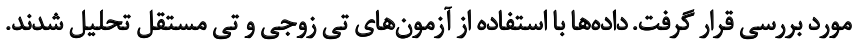

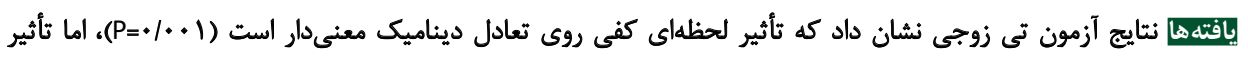

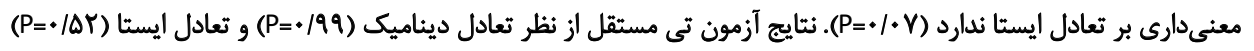

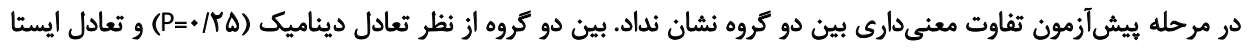
(P=-/Mr)

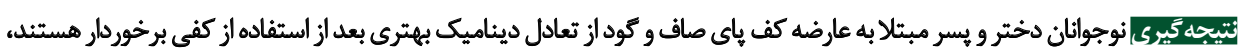

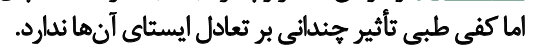

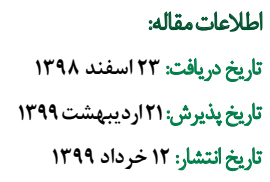

فعاليت عضلانى و استرس وارد بر ساختارهاى سمت داخل باء

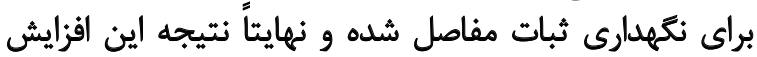

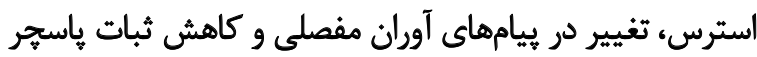

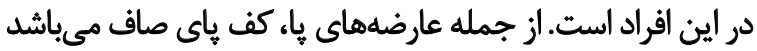

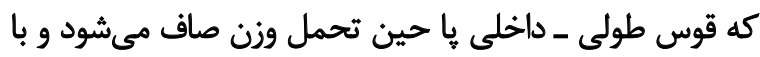

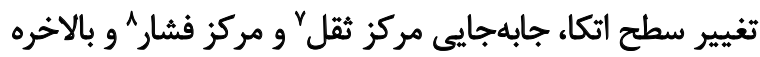

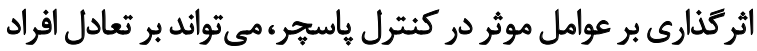

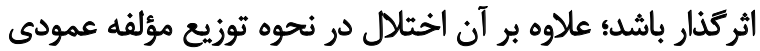

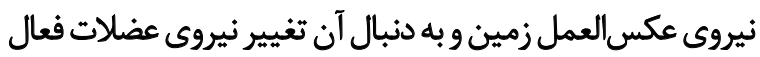

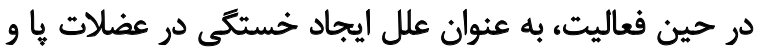

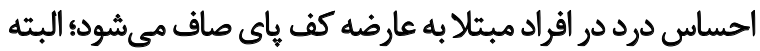

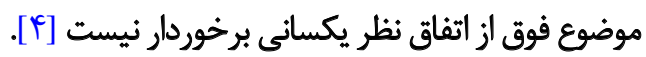

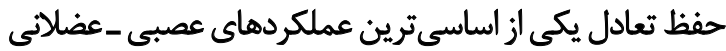

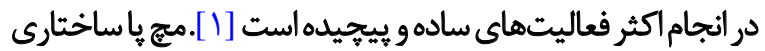

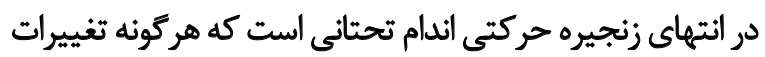

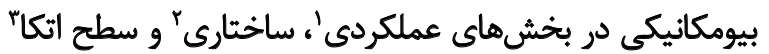

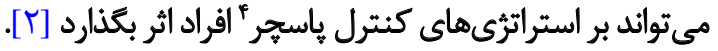
قوسهاى كف بايى نقش بسرايى در تعديل و توزيع فشار

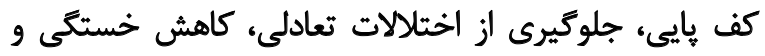

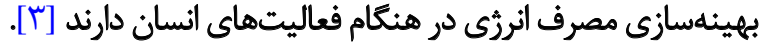
كاهش تجانس مفاصل ميدتارسال ه و ساب تالار ه سبب افزايش

كف بايى انسان با سطح اندك خود، نقش مهمى دي در حفظ تعادل

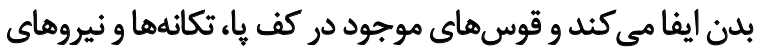
وارده از زمين را جذب و تعديل ميىكنيند [ه]. بر اساس تئورى هو

2. Structural

3. Base of spurt

4. Pasture

7. Center of Gravity (COG)

5. Mid tarsal joint

8. Center of Pressure (COP)

6. Subtalar Joint

*نويسيده مسينول: زهراكريلى نوين نشائى: تهران، واحد تهران مركزي، دانشكاه آزاد اسلامي، دانشكده تربيتبدنى و علوم ورزشى، كروه بيومكانيك ورزشى. تلفن: يست الكترونيكى: zahra koreili@yahoo.com 


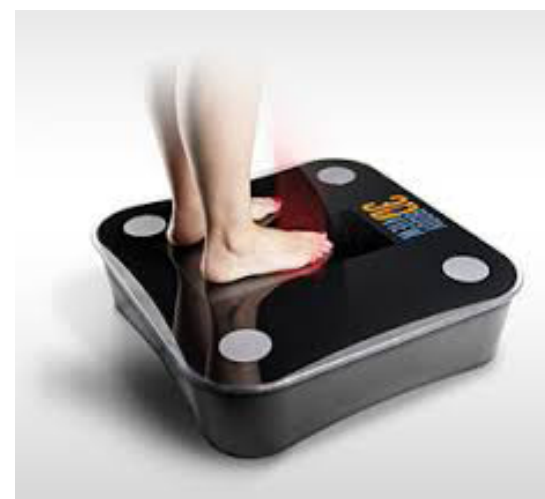

مجله بيومكانيك وزنث

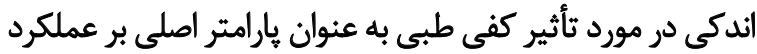

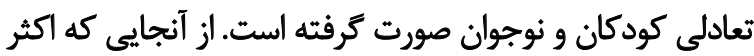

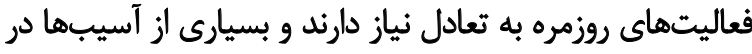

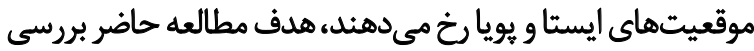

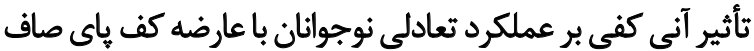

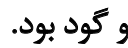

$$
\text { روشُشَّاسي }
$$

تحقيق حاضر از نوع توصيفى، نيمهتجربى و كاربردى است كه

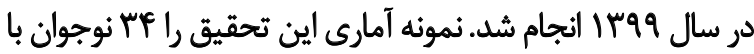

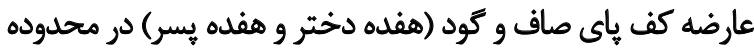

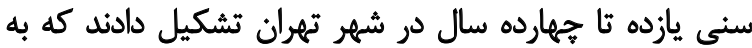

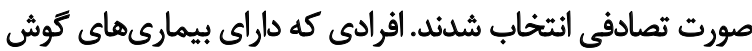

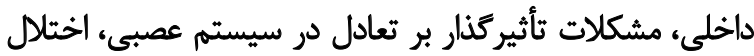

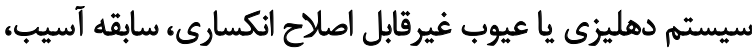

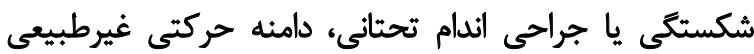

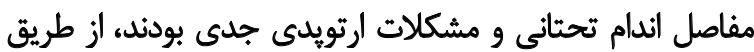

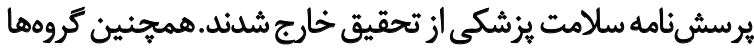

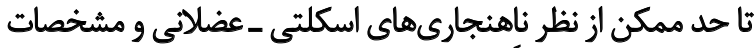

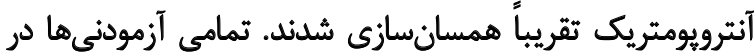

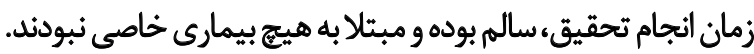

\section{Q78}
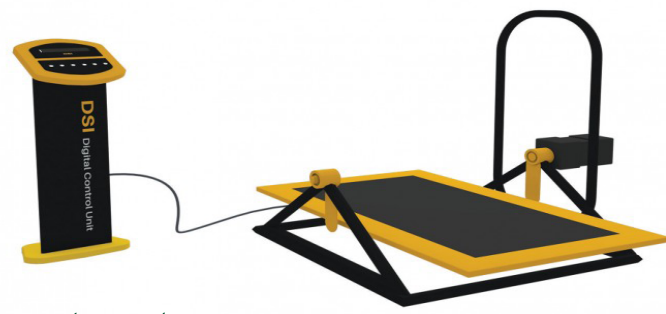

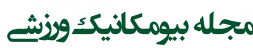

تصوير Y. دسثكاه تعادل يويا CNE (فرمول امتياز - زمان حفظ تعادل تقسيم

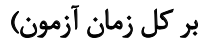

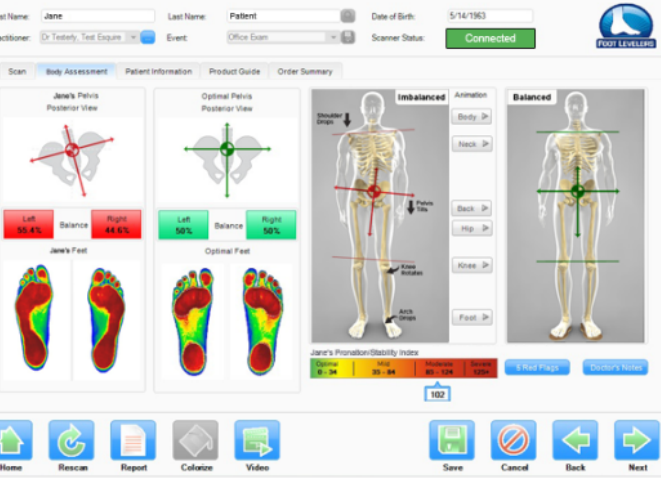

تصوير ا. فوت اسكنر3D BODU VIEW وخروجى دستكاه

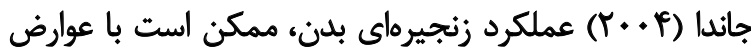

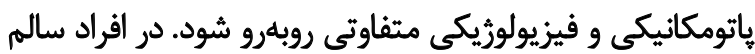

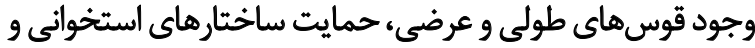

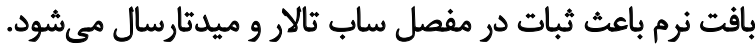

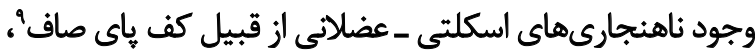

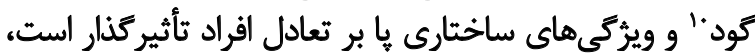

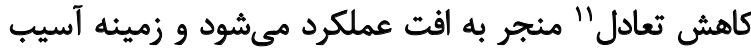

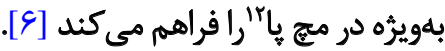

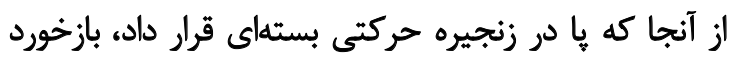

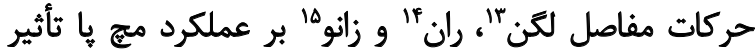

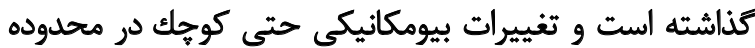

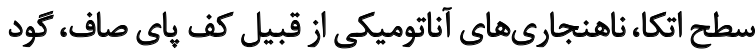

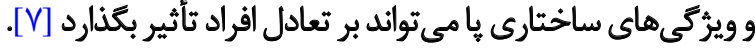

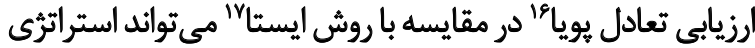

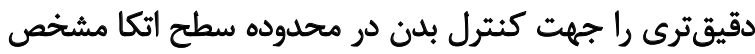

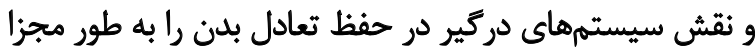

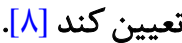

مرور تحقيقات كذشته نشاندهنده ارزيابى و بروريى بارئ

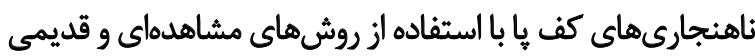

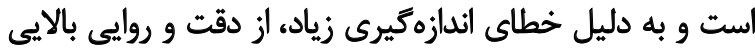

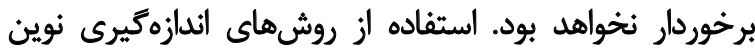

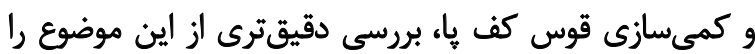

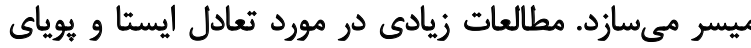

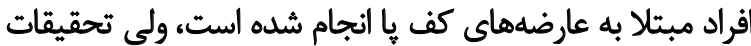

9. Flat foot

10. Pes cavus

11. Balance

12. Ankle joint

13. Hip

14. Femur

15. Knee

16. Dynamic balance

17. Static balance 
نتايج

ميانكين شاخص هاى جمعيتشناختى آزمودنى إنها براى سن

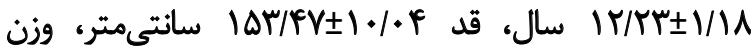
طاي طول ياى راست

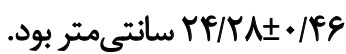

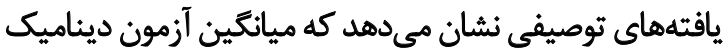

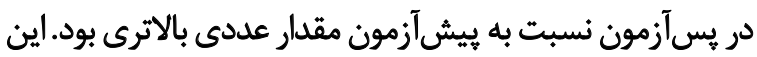

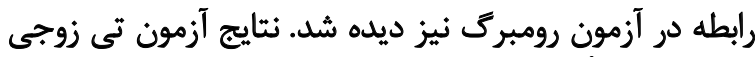

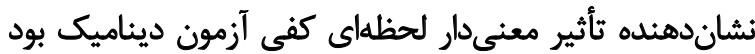

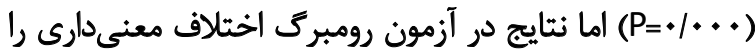

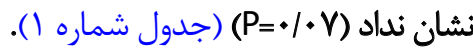
كتايج آزمون تي زوجى به منظور تأثير آنى كفى بر دو دو كروه

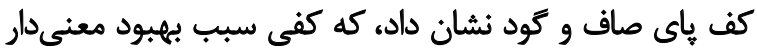

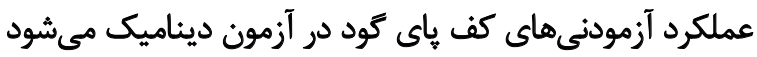

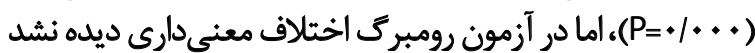

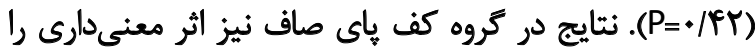

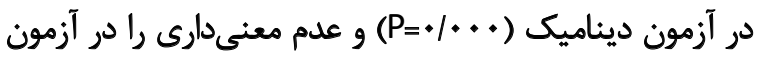

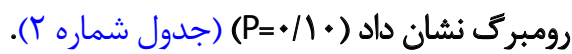

نتايج آزمون تى مستقل نيز به منظور مقايسه دو كروه كف بِاى

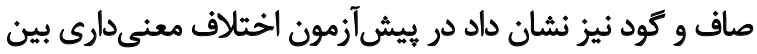

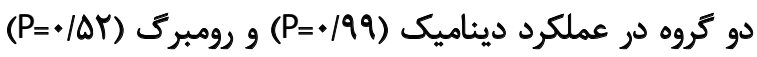

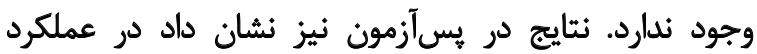

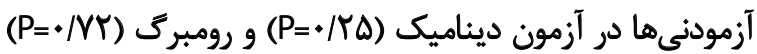
اختلاف معنى دارى وجود ندارن (جدول شماره س)

بحث

هدف از تحقيق حاضر بررسى تأثير لحظهاى كفى بر بر روى بادي

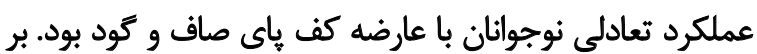

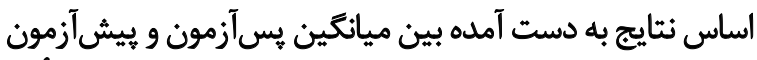

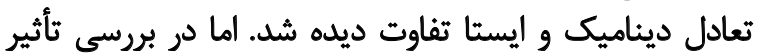

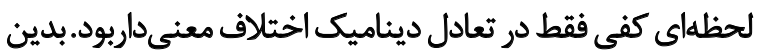

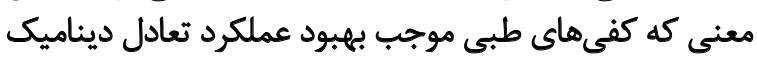

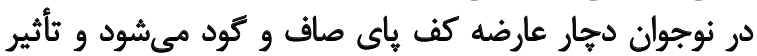
جندانى بر روى تعادل ايستا آنها ندارد.

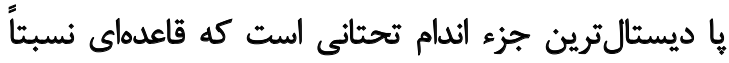

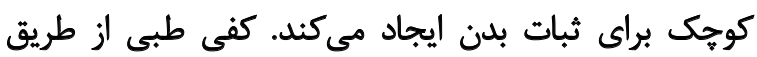

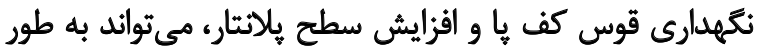

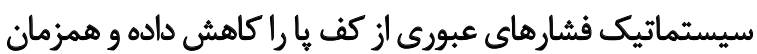

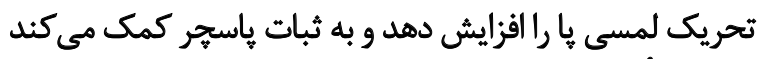

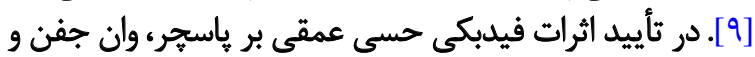

براى اندازهيرى قد از قدسنج ديوارى مثلثى (26SM) و براى

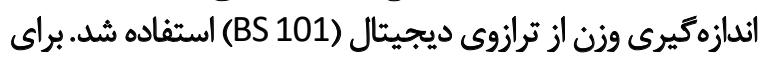

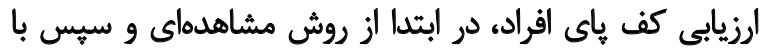

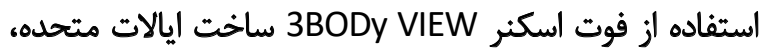

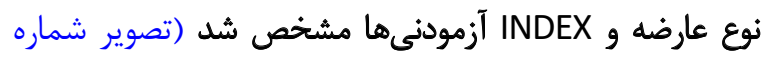

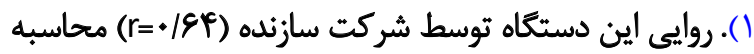

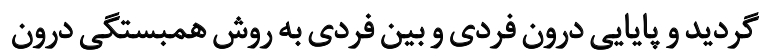

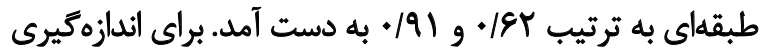

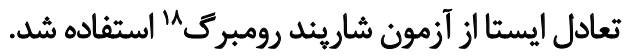

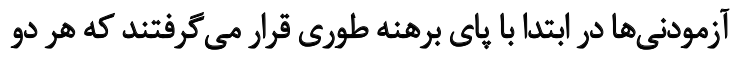

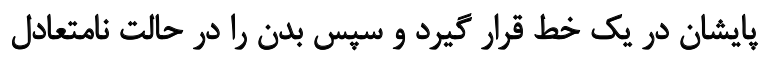

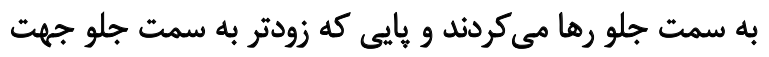

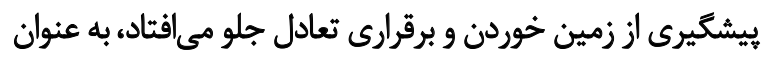

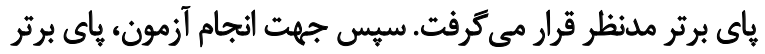

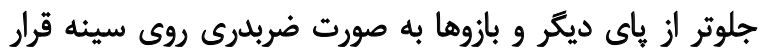

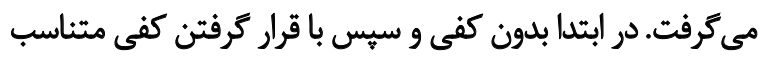

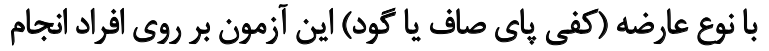

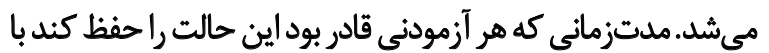

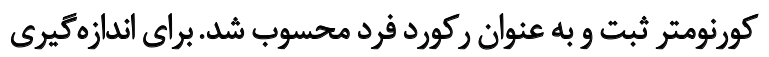

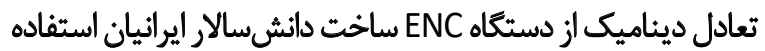

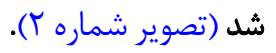

اين دستًاه داراى دو بخش سختافزارى (يك بخش ويرٔه ايستادن

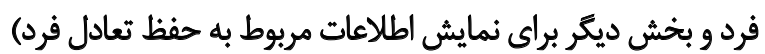

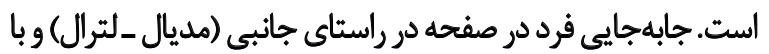

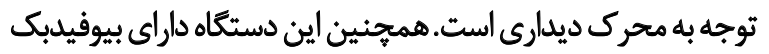

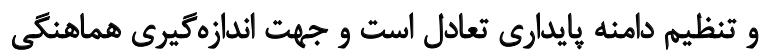

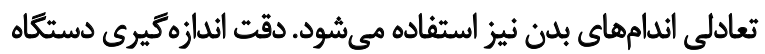
Dynamic Balance

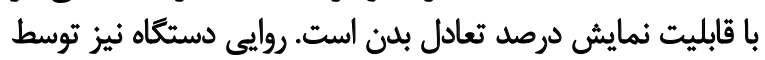

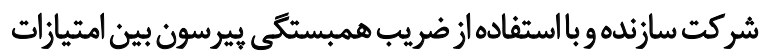

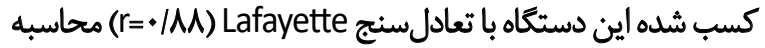

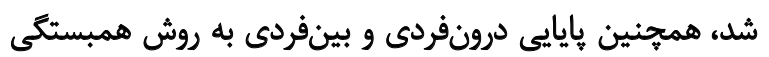

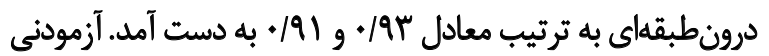

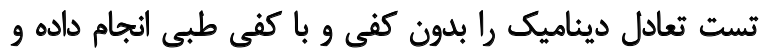

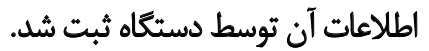

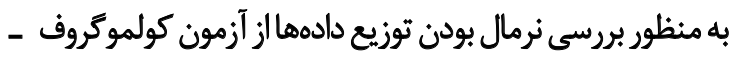

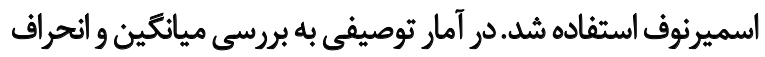

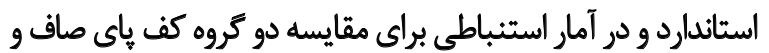

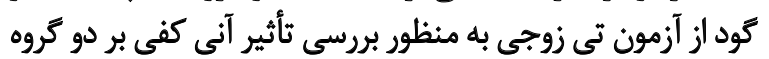

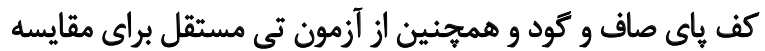

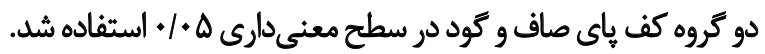

18. Sharpened-Romberg Test 
جدول ا.نتايج آزمون تى زوجى به منظور بررسى اثر آنى كفى بر عملكرد تعادلى آزمودنىها

\begin{tabular}{|c|c|c|c|c|c|}
\hline \multirow{2}{*}{ سطح معنى دارى } & \multirow{2}{*}{$\mathbf{t}$} & \multirow{2}{*}{ اختلاف ميانكين } & \multicolumn{2}{|c|}{ ميانكين+انحراف استاندارد } & \multirow{2}{*}{ شاخص } \\
\hline & & & يسأزمون & يششآزمون & \\
\hline.+ & $-1 \cdot / A f$ & - & 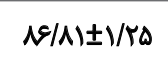 & ex/RtIV/QI & ديناميك \\
\hline.$/ * V$ & $-1 / 1 \Delta$ & $-1 / r \mu$ & $19 / 49 \pm .194$ & $W \cdot \Delta \pm \cdot / 8$ & رومبرى \\
\hline
\end{tabular}

مجلله بيومكانيك ورنش

جدول Y. نتايج آزمون تى زوجى به منظور بررسى اثر آنى كفى بر عملكرد تعادلى آزمودنىها در دو كروه كف باى كود و صاف

\begin{tabular}{|c|c|c|c|c|c|c|}
\hline \multirow{2}{*}{ سطح معنى دارى } & \multirow{2}{*}{$\mathbf{t}$} & \multirow{2}{*}{ اختلاف ميانكين } & \multicolumn{2}{|c|}{ ميانكين+انحراف استاندارد } & \multirow{2}{*}{ شاخص } & \multirow{2}{*}{ تروه } \\
\hline & & & يس آزمون & ي يش آزمون & & \\
\hline$\%$ & $-V / A r$ & $-M N A Y$ & $A T / Q I \pm N / T$ & $8 \Delta / * A_{ \pm} V / q \mu$ & ديناميك (IT) & \multirow{2}{*}{ كف باى كود } \\
\hline.$/ 4 T$ &.$- / A Y$ & $-1 / 18$ & $19 / V \Delta_{ \pm} \Psi / 9 \Psi$ & $\mathbb{N Q A} \pm T / F T$ & روميرى (IT) & \\
\hline$\%$ & $-s / N$ & $-r T / R Y$ & $A V / \Psi \bar{I} I / q V$ & $\varepsilon_{\Delta} / \cdot F_{ \pm} \pm \mid r / \tau$. & ديناميك (MT) & \multirow{2}{*}{ كف باى صاف } \\
\hline.$/$ & $-1 / 89$ & $-1 / \Delta$ & $1 V / r_{ \pm} r / 9 F$ & $|Y M \pm+r / 9|$ & رومبرى (TM) & \\
\hline
\end{tabular}

مجله بيومكانيك:وزنش

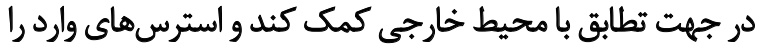

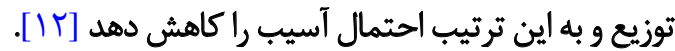
نتايج يروهش حاضر با تحقيق كريبل و همكاران كه اظهار

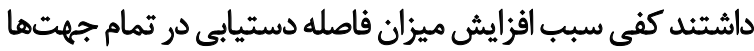

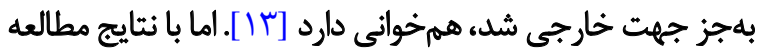

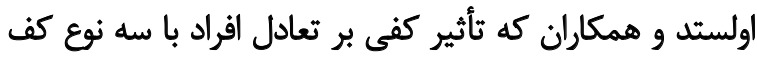

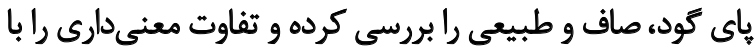

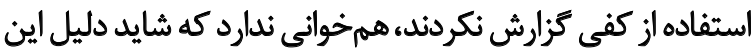

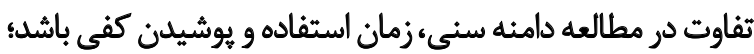

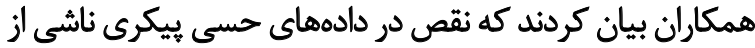

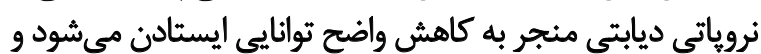

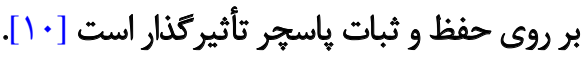

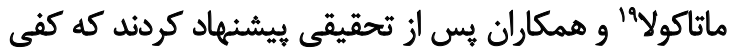

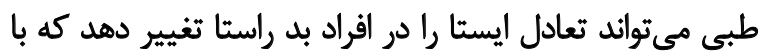

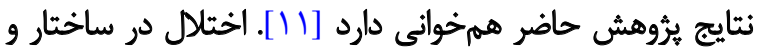

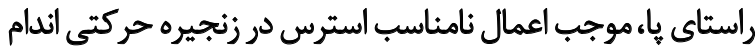

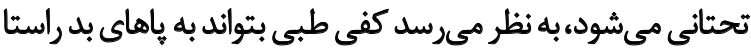

19. Matacola

جدول r. نتايج آزمون تى مستقل به منظور مقايسه عملكرد آزمودنىها در دو كروه كف ياى كود و صاف

\begin{tabular}{|c|c|c|c|c|c|c|c|}
\hline معنى سطح & $t$ & $\mathbf{F}$ & اختلاف ميانكين & ميانكين+|انحراف|ستاندارد & 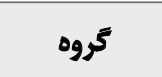 & شاخص & مرحله \\
\hline.$/ 99$ & $\%$ & $1 / .1$ & $\%$ & $\begin{array}{l}q \Delta / \cdot \wedge_{ \pm} q / q \Psi \\
q \Delta / \cdot \varphi_{ \pm} \mid r / \psi\end{array}$ & كف كف باي كَّد & ديناميك & \multirow{2}{*}{ إييشآزمون } \\
\hline . $/ \Delta r$ & "ז & $\%$ & $\cdot(A)$ & $\begin{array}{l}W Q A_{ \pm} r / F T \\
\left|W / W_{ \pm} r / Q\right|\end{array}$ & كف بإى كود & رومبرى & \\
\hline.$/ 2 \Delta$ & $-1 / 19$ & $\%$ & $-r / \varphi \cdot$ & 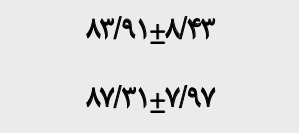 & كف بف باى كود & ديناميك & \multirow{2}{*}{ بس أزمون } \\
\hline.$M r$ & ه & $.1 . \Delta$ & . Mr & $\begin{array}{l}19 / V \Delta_{ \pm} r / q \Psi \\
19 / T V_{ \pm} r / q 4\end{array}$ & كف بائ كود باي صاف & رومبرى & \\
\hline
\end{tabular}

مجله بيومكانيكورزنش 
كودكان با كف بايى صاف و طبيعى يرداختند و بيان كردند تعادل

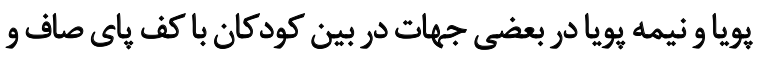

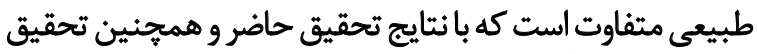

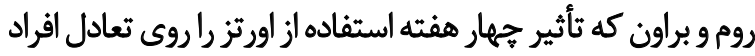

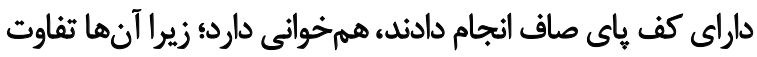

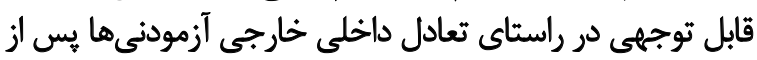

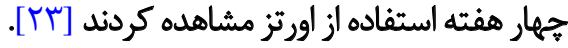

نكته قابل توجه اين است كه در بررسى ادبيات نوع ساختار

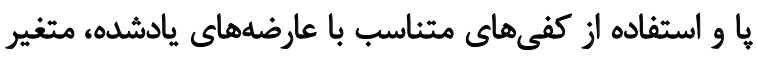

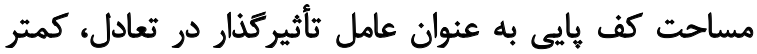

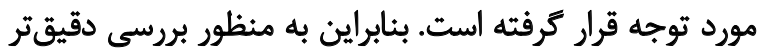

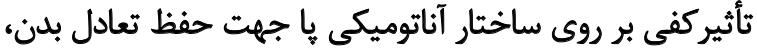

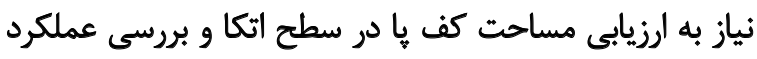
عضلات قبل و حين استفاده از كفى، ضرورى به نظر ميرسد.

\section{نتيجه تيرى نهايى}

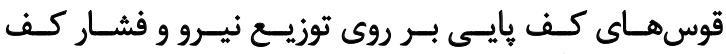

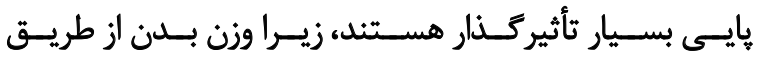

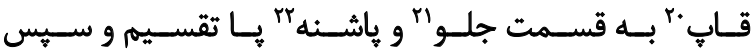

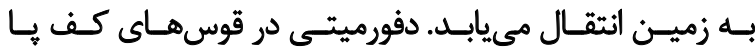

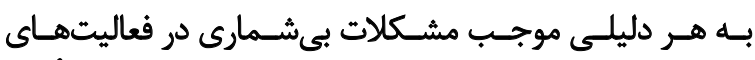

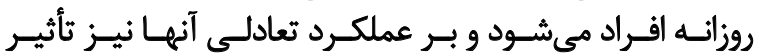

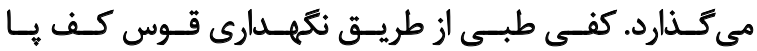

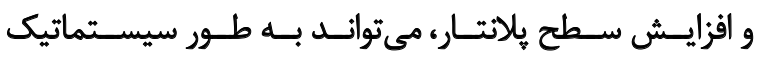

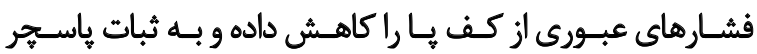

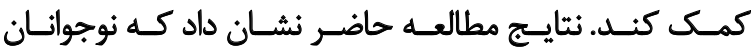

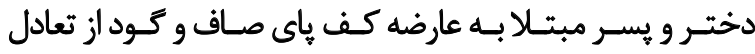

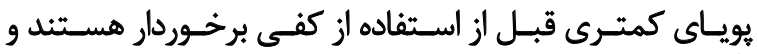

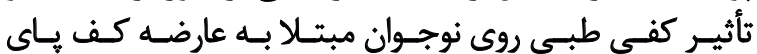

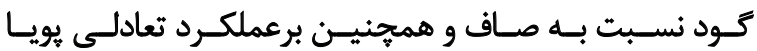

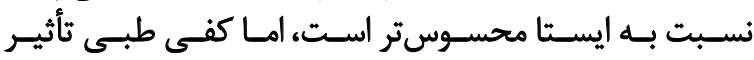

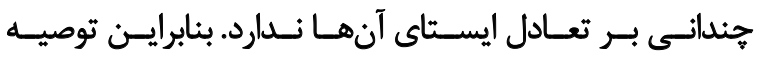

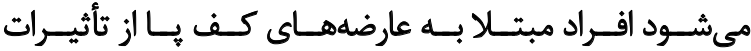

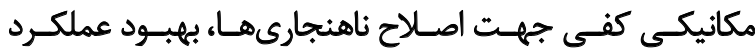

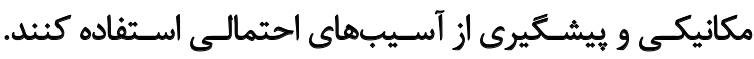

ملاحظات اخلاقي

$$
\text { بيروى از اصول اخلاق يُوهش }
$$

تمامى مراحل يُروهش با رعايت اصول اخلاقى و با اخذ

20. Talus

21. Meta tarsal

22. Calcaneuse

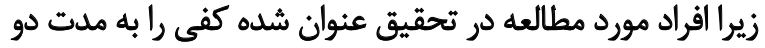

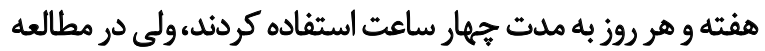

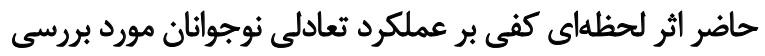

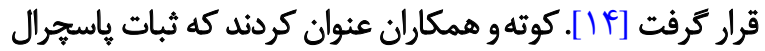

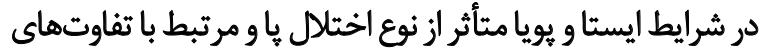

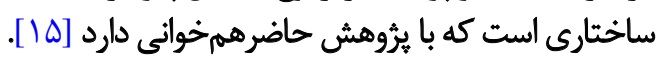
كارن و همكاران تعادل ايستاو يوياي افراد را با استفاده از سيستم

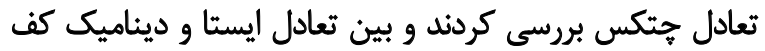

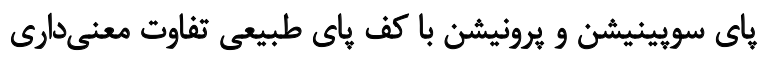

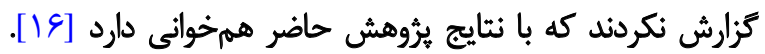

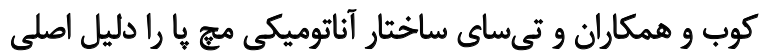

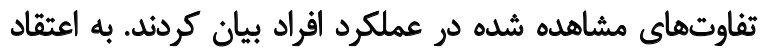

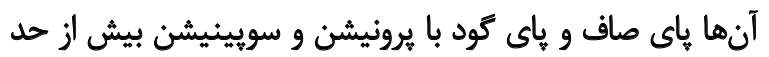

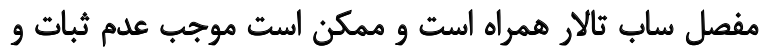

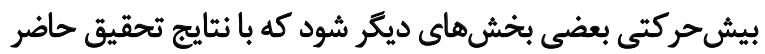

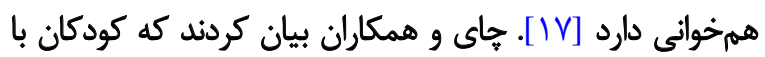

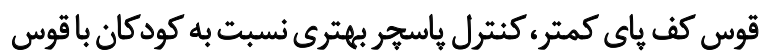

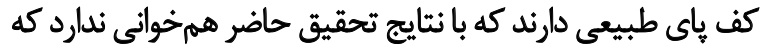

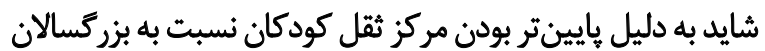

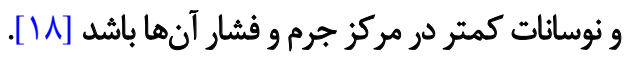

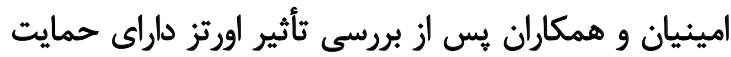

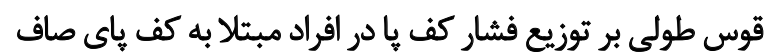

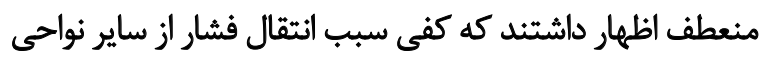

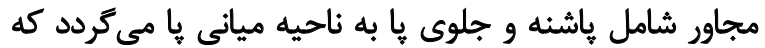

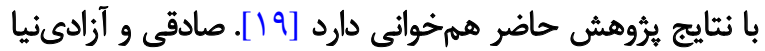

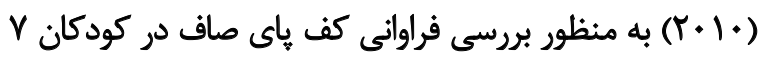

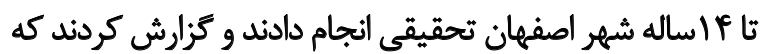

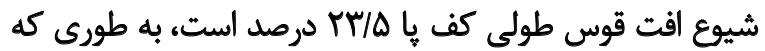

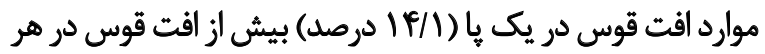

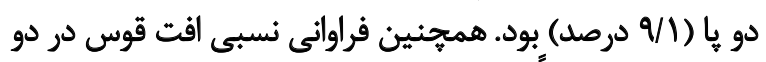

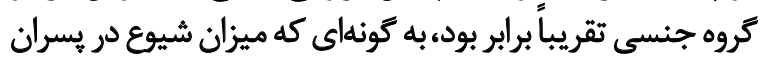

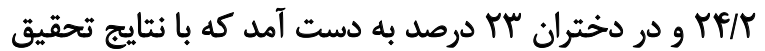

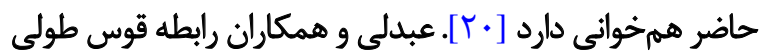

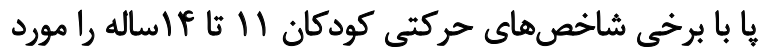

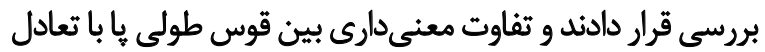

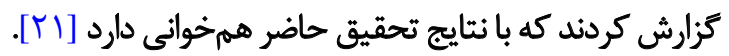
بايهدار و همكاران با استفاده از دستًاه تعادلسنج بايودكس كانس

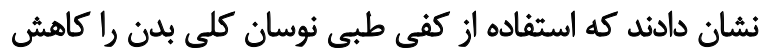

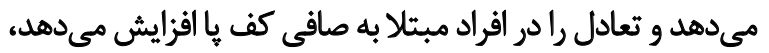

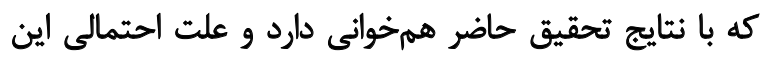

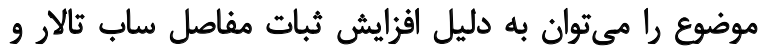

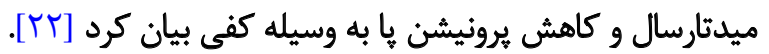

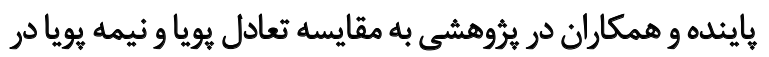




$$
\text { رضايتنامه كتبى از شركت كنيدكان انجام شد. }
$$

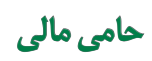

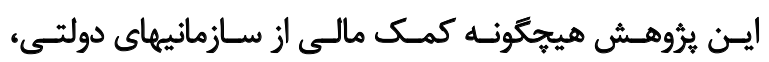

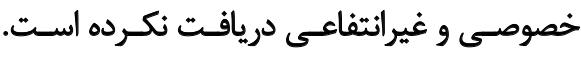

$$
\text { مشاركت ثويسندكان }
$$

تمامى نويسندكان در نكارش اين مقاله مشاركت داشتند.

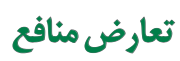

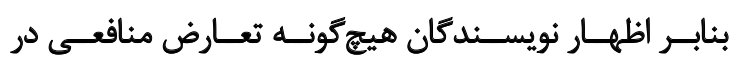

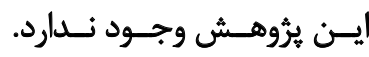

$$
\text { تشكر و قدردانى }
$$

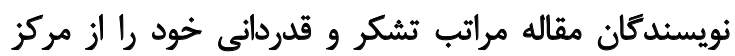

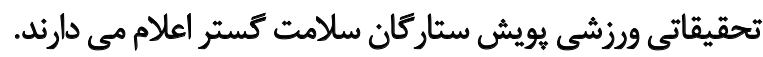




\section{References}

[1] Hosseini R, Norasteh AA, Nemati N. Comparing the balance of male athletes aged 11-14 years with and without genu varum. J Sport Biomech. 2019; 4(4):54-65. [DOI:10.32598/biomechanics.4.4.54]

[2] Aminian G, Safaeepour Z, Farhoodi M, Pezeshk AF, Saeedi H, Majddoleslam $B$. The effect of prefabricated and proprioceptive foot orthoses on plantar pressure distribution in patients with flexible flatfoot during walking. Prosthet Orthot Int; 2013; 37(3):227-32. [DOI:10.1177/0309364612461167] [PMID]

[3] Fan Y, Fan Y, Li Z, Lv C, Luo D. Natural gaits of the non-pathological flat foot and high-arched foot. PloS One. 2011; 6(3):e17749. [DOI:10.1371/ journal.pone.0017749] [PMID] [PMCID]

[4] Cobb SC, Tis LL, Johnson BF, Higbie EJ. The effect of fore foot varus on postural stability. J Orthop Sports Phys Ther. 2004; 34(2):79-85. [DOI:10.2519/jospt.2004.34.2.79] [PMID]

[5] Bonnie Y, San T BD, Yu BF, Boone DA, Mphil CP. Quantitative comparison of plantar foot shape under different weightbearing condition. J Rehabil Res Dev. 2000; 40:526-628. [PMID]

[6] Ghasemi V, Rajabi R, Alizadeh M, Dashti Rostami K. [Comparison of dynamic balance in males with diffrent foot types (Persian)]. Sport Medicine; 2011; 3(1):5-20. https://jsmed.ut.ac.ir/article_24187.html

[7] Gribble PA, Hertel J. Considerations for normalizing measures of the Star Excursion Balance Test. Measure Physic Educe Exerts Sci. 2003; 7(2):89-100. [DOI:10.1207/S15327841MPEE0702_3]

[8] Emery CA, Cassidy JD, Klassen TP, Rosychuk RJ, Rowe BB. Development of a clinical static and dynamic standing balance measurement tool appropriate for use in adolescents. Phys Ther. 2005; 85(6):502-14. [DOI:10.1093/ptj/85.6.502] [PMID]

[9] Hamlyn C, Docherty CL, Klossner J. Orthotic intervention and postura stability in participants with functional ankle instability after an accommodation period. J Athl Train. 2012; 47(2):130-5. [DOI:10.4085/10626050-47.2.130] [PMID] [PMCID]

[10] Van Geffen JA, Dijkstra PU, Hof AL ,Halbertsma JPK, Postema K. Ef fect of flat insoles with different Shore A values on posture stability in diabetic neuropathy. Prosthet Orthot Int. 2007; 31(3):228-35. [DOI:10.1080/03093640600994557] [PMID]

[11] Mattacola CG, Dwyer MK, Miller AK, UhI TL, McCrory JL, Malone TR. Effect of orthoses on postural stability in asymptomatic subjects with rear foot malalignment during a 6-week acclimation period. Arch Phys Med Rehabil. 2007; 88(5):653-60. [DOI:10.1016/j.apmr.2007.02.029] [PMID]

[12] Kido M, Ikoma K, Hara Y, Imai K, Maki M, Ikeda T, et al. Effect of therapeutic insoles on the medial longitudinal arch in patients with flatfoot deformity: A three-dimensional loading computed tomography study. Clin Biomech. 2014; 29(10):1095-8. [DOI:10.1016/j.clinbiomech.2014.10.005][PMID] [PMCID]

[13] Rome K, Brown CL Randomized Clinical trail into the impact of rigid foot orthoses on balance parameters in exercise sively pronated feet. clinical rehabilitation. 2004; 18(6):624-30. [DOI:10.1191/0269215504cr767oa] [PMID]

[14] Olmsted LC, Hertel J. Influence of foot type and orthotics on static and dynamic postural control. J Sport Rehabil. 2004; 13(1):54-66. [DOI:10.1123/jsr.13.1.54]

[15] Cote KP, Brunet ME, Gansneder BM, Shultz SJ. Effects of pronated and supinated foot postures on static and dynamic postural stability. J Athl Train. 2005; 40(1):41-6. [PMCID]
[16] Karen PC, Michael EB, Bruce M, Gansneder and Sandra JS. Effect of pronated and supinated foot posture on static and dynamic postural stability. Journal Athlete Train. 2005; 40:41-6. [PMID] [PMCID]

[17] Tsai L-C, Yu B, Mercer VS, Gross MT. Comparison of different structura foot types for measures of standing postural control. J Orthop Sports Phys Ther. 2006; 36(12):942-53. [DOI:10.2519/jospt.2006.2336] [PMID]

[18] Lin C-H, Lee H-Y, Jason Chen J-J, Lee HM, Kuo MD. Development of a quantitative assessment system for correlation analysis of footprint parameters to postural control in children. Physiol Meas. 2006; 27(2):119 30. [DOI:10.1088/0967-3334/27/2/003] [PMID]

[19] Aminian G, Farhoodi M, Safaeepour Z, Farjad Pezeshk A. [The assessment of the effect of longitudinal arch support insole on plantar pressure distribution in subjects with flexible flatfoot (Persian)]. Iran J War Public Health. 2012; 4(16):43-8. http://ijwph.ir/article-1-226-fa.htm

[20] Sadeghi E, Asadinia F. [To determine the prevalence of flat foot in children 7 to 14 years in Isfahan (Persian)]. Journal of Medical Council of Iran. 2010; 29(2):142-9. http://jmciri.ir/article-1-1423-fa.html

[21] Abdoli B, Taymoori M, Zamani Sani SH, Zeraat Kar M, Hovnloo F. [Relationship between Plantar longitudinal arches and Some Selected Motor Parameters in Children aging 11 to 14 years (Persian)].JRRS. 2011 7(3):381-90. [DOI: 10.22122/jrrs.v7i3.209]

[22] Payehdar S, Saeedi H, Ahmadi A, Kamali M, Mohammadi M, Abdollah V. Comparing the immediate effects of UCBL and modified foot orthoses on postural sway in people with flexible flatfoot. Prosthet Orthot Int. 2016; 40(1):117-22. [DOI:10.1177/0309364614538091] [PMID]

[23] Payandeh M, Yazi N, Ebrahimi A, Damavandi M, Ameli M, Safari M. [Comparison of Dynamic and Semi dynamic Balance of Flat Foo with a Normal Foot (Persian)]. J Zabol Univ Med Sci Health Serv. 2015; 7(2):2. https://www.researchgate.net/publication/342864512_ 